\title{
Supervised Image Classification of Chaos Phenomenon in Cumulonimbus Cloud Using Spectral Angle Mapper
}

\author{
Wanayumini $^{\mathrm{a}, 1}$, Opim Salim Sitompul ${ }^{\mathrm{b}, *}$, Saib Suwilo ${ }^{\mathrm{c}}$, Muhammad Zarlis ${ }^{\mathrm{a}, 2}$ \\ ${ }^{a}$ Department of Computer Science, Universitas Sumatera Utara, Medan Indonesia \\ E-mail: ${ }^{1}$ wanayumini@gmail.com; ${ }^{2}$ m.zarlis@usu.ac.id \\ ${ }^{b}$ Department of Information Technology, Universitas Sumatera Utara, Medan Indonesia \\ E-mail: "opim@usu.ac.id (corresponding author) \\ ${ }^{c}$ Department of Mathematics, Universitas Sumatera Utara, Medan Indonesia \\ E-mail:saib@usu.ac.id
}

\begin{abstract}
In the field of remote sensing, in addition to the weather forecast, atmospheric dynamics, oceans, cloud cumulonimbus, and Tornado are part of the phenomenon of chaos. Because in the clouds cumulonimbus, there are some layers with a gray border indicating irregular and uncertain. There is a boundary line on the layers of Cumulonimbus Clouds that could be identified based on the pixel where the differences in the intensity values are extremes. A cloud layer cumulonimbus with a gray edge border can be used as the basis for predicting the occurrence of a tornado based on a pixel location that has specific characteristics. In this research, a Supervised Image Classification algorithm with Spectral Angle Mapper was performed to get the minimum and maximum pixel intensity interval values based on spectral angles in cumulonimbus clouds. Spectral angles allow for quick mapping in determining the spectral similarities between two spectrums on cumulonimbus cloud layers. The spectral similarities are calculated by referring to the angle between the spectral forming the same dimensional vector space on the RGB color spectrum. Early detection in cumulonimbus cloud layers will indicate the occurrence of chaos phenomenon, which could be used to predict tornadoes. The results showed that the Spectral Angle Mapper approach gave minimum and maximum pixel intensity values interval of the Average Correlation Angle in the dataset image Cumulonimbus Cloud with a classification accuracy value of $95.83 \%$.
\end{abstract}

Keywords—cumulonimbus clouds; tornados; chaos phenomenon; spectral angle mapper; average correlation angle.

\section{INTRODUCTION}

The Cumulonimbus Cloud is one of the earliest formations of a tornado, wherein the clouds there is a phenomenon that shows irregularities and cannot be prepopulated. This phenomenon of irregularities is then called chaos [1]. The tornado is the wind caused by the impact of the cumulonimbus cloud, which usually grows during the rainy season [2], [3]. Nevertheless, to ensure that a chaotic phenomenon will lead to the occurrence of a tornado, some feature characteristics should be determined, as have been suggested in many related research works.

Determining the characteristics that indicated the potential occurrence of the wind of the nipple is difficult because the incident always occurs in the sky. One way to get those features is through satellite imagery, for example, the extreme heat difference is a character by a present of a Cumulonimbus Cloud that has a grey border boundary that exceeds the rule limit in a stamped image with color intensity [3], [4]. The color intensity or color quality of the image is the difference in brightness and the gloom of a color. The color image can be used as the basis for parametric related to the initializing condition of the tornado [3], [5]. Imagery has a pixel that is used as the basis for determining the sharpness of an object [6]. Pixel optimization is useful for object detection, and the segmentation of pixel values is considered a significant factor.

The rapid intensification of tropical cyclones forms a formation called eye formation, which is essential information in the prophecy of Storm [7]. Ant Colony Optimization (ACO) algorithm is used to obtains a spectral, identifiable image pixel that can be provided to add solutions to field sample collection [8], [9]. The method is used to reconstruct a spectral dataset into phases, where the hidden chaos characteristics in the dataset can be rearranged into predictable sequences. Thus, better end members can be selected from a spectral or hyperspectral dataset. 
Some studies researched high-resolution remote sensing imagery with the supervised segmentation method; the method enhances the expression of uncertainty for pixel membership [6], [9], [10]. Other studies observe the storm, using satellite-based techniques such as Tropical Analysis and Forecast Branch, Analysis of satellite imagery with Dvorak Advanced techniques, or abbreviated to ADT used by the institute or university of Wisconsin-Madison [2], [11]. Satellite imagery data from the National Oceanic and Atmospheric Administration and the National Aeronautics and Space Administration are commonly used for weather forecasts, including tropical cyclones, storm, and rainfall [12]-[14].

The Supervised Classification method was used with the Supervised Image Classification model through the image segmentation process using an Edge detection algorithm and Next to get the prediction value by using Spectral Angle Mapper (SAM) algorithm [11], [15], [16]. The model is proposed because it is relatively simple, which provides predictive value and uncertainty. The predicted value can from the process result through the Spectral Angle Mapper Classification algorithm in the form of the minimized and maximum pixel value intervals based on the Average Correlation Angle on the RGB color spectrum.

Average Correlation Angle is the average angle of correlation between variable $\mathrm{x}$ and variable $\mathrm{y}$ on the color spectrum of cloud images cumulonimbus and tornado. Both variables can be correlated if there is a change between one variable and the other basis on a regular in the same direction. Tornadoes and clouds cumulonimbus have some free or independent variables. A free variable is a variable that can affect other variables. Among other variables generated by Tornado and cumulonimbus clouds is the intensity of light, where the intensity of the light cannot be arranged by humans [17], [18].

\section{MATERIALS AND METHODS}

In conducting the collection and retrieval of data used as a dataset to process the analysis with the supervised image classification method, this research uses data contained in the Google image of good that comes from the satellite NASA through NASA [19], [20]. The dataset in the analysis is taken from the image dataset Cumulonimbus cloud, which is the beginning of the occurrences of a tornado-like, the one found in Figure 1.

Furthermore, the process of extracting irregular patterns contained in the layer cloud cumulonimbus using an edge detection algorithm, as seen in Figure 6. The finals process is then done to get the minimized and maximum pixel value of the average correlation angle by using the Spectral Angle Mapper algorithm to get the classifications of supervised images (supervised image classification). The dataset used with this method is a dataset image cumulonimbus cloud as many as 24 datasets in figure 1 .

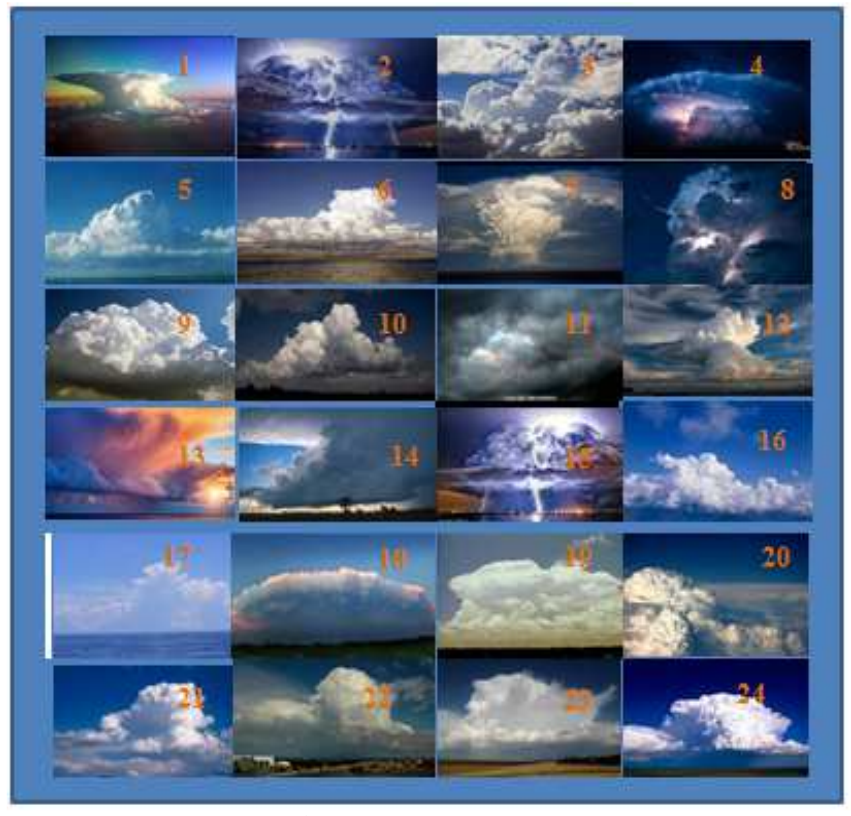

Fig.1 RGB Image Dataset

\section{A. Spectral Angle Mapper (SAM) Classification}

Figure 2. It is a graph of the Spectral Angle Mapper (SAM) method based on spectral angles. The spectral angle is the angle between the color spectrum in the $\mathrm{x}\left(\lambda_{1}\right)$ variable and the $\mathrm{y}\left(\lambda_{2}\right)$ variable as the vector in the dimensions space equal to the number of bands/channels. The processing result of the method is the ASCII code.

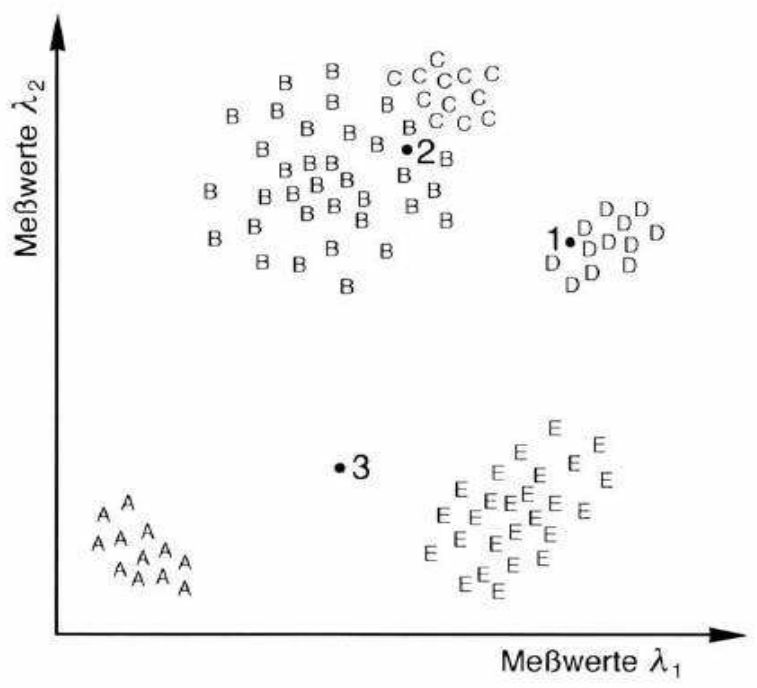

Fig.2. SAM Classification method

It is a pixel-based spectrum classification that uses dimensional-n angles to match pixels to training data. [21] and [19] Spectral angle mapper method is to determine the spectral similarity between two spectra by calculating the angle between the spectral as a vector in a space with the same dimension. Spectrum the color in this case uses is a color RGB or Red Green Blue. 
The classification of SAM is a direct method of automatic by comparing the spectral image of the cloud with the image of a tornado. This method is not sensitive to lighting because the SAM algorithm only uses the vector direction instead of the vector length. The SAM classifications result is an image that shows the best fit for each pixel [22].

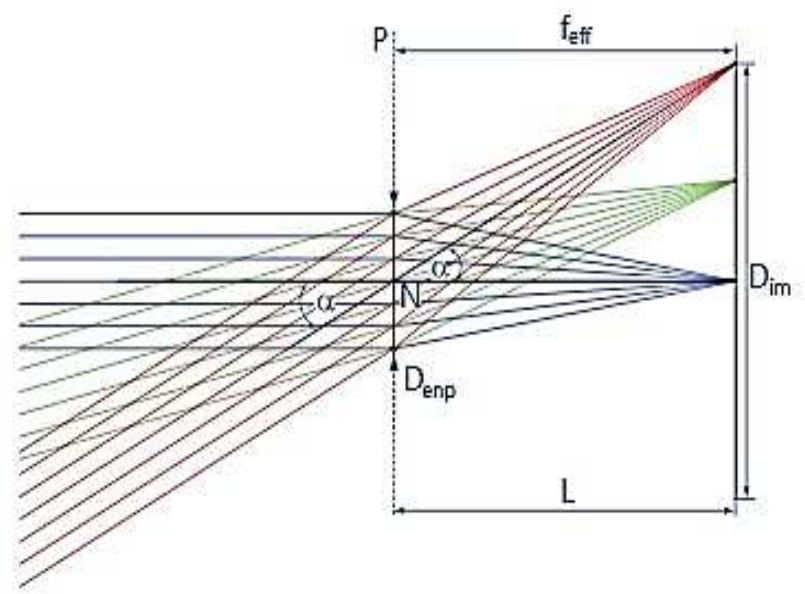

Fig.3 Direction Vector SAM algorithm

Figure 3 is an illustration or overview of the result classifications with the SAM method. The reflected light formed on the RGB color spectrum resulted in a spectral angle $(\alpha)$ via vector direction in the $\mathrm{X}(\mathrm{N})$ variable and the $\mathrm{Y}$ (P) variable. $D_{\text {im }}$ is an image circle diameter, $D_{\text {eep }}$ is a diameter for the determined of spectral angles.

As for the direction of the vector generated on the cloud, dataset image and the tornado generates the average correlation angle value. Average Correlation Angle is the mean angle of correlation, where the angle of correlation is the analysis technique in the statistics that is the angle that has the relationship between two variables or degrees of relationship or degree of association e.g. variable $\mathrm{X}$ and variable $\mathrm{Y}$. These variables are to correlate when there is a change to a variable that one will be following by a change in another variable regularly in the same direction (positive correlation) or opposite (negative correlation).

In this case, on the Tornado wind and the clouds cumulonimbus, some variables change and tend to be beyond human control. Wind Tornado and clouds cumulonimbus do not consider the intensity of the resulting light but based on the vector direction. Therefore, the Spectral Angle Mapper method is used to determine the vector direction found on the cumulonimbus cloud layer. Where the layers in the clouds cumulonimbus there are vector directions in the same dimensional space as the number of bands.

Spectral Angle Mapper (SAM) is a good method of classifications, as it evaluates for the analysis of spectral similarities to bring out the target reflection of the characteristics of the cloud image cumulonimbus. The classifications of Cumulonimbus Cloud using Spectral Angle Mapper (SAM) algorithm is performs with equation formula:

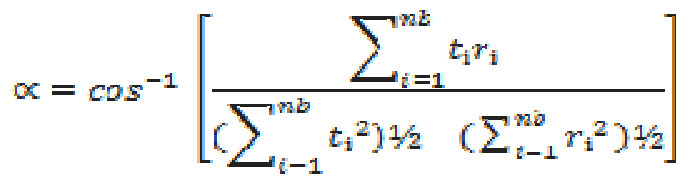

Where:

$\mathrm{Nb}-$ : number of bands (channels) in the image

t- : Spectral pixels

r- : Spectral reference (measurement spectral library)

$\alpha-$ : Spectral angle.

\section{B. Classifications of Imagery (Image Classification)}

Remote sensing technology can monitor the existence of a phenomenon that can change information on a regular and practical basis, as well as improve the classifications of images accurately. Image classification is a form of classifications that is useful in monitoring against remote sensing data [23], [24]. In the classification of remote sensing, images generally consists of the classifications of unsupervised images and the classifications of images supervised. Unsupervised image classification method does not need an understanding of the field of study, because it will save time and money. In some instant applications, the accuracy of the image classifications does not result in shapes that follow the rules.

The classification method of the supervised image has a good accuracy value [25], [26]. The value of accuracy used can meet the average value of a sample area that starts at the core of the cluster without any other consideration. The process was conducted by adding the Chaos method in performing the process of searching the data with genetic algorithms [27], [28]. The process is performed to provide solutions in improving the accuracy value and rapid search of the classifications of remote sensing images based on the initial sensitive state of a chaotic system and parametric system [29], [30].

\section{Image Classification Techniques}

A hybrid method was used to combine more than one classifications method supervised by unsupervised methods [19], [31]. The method is often used in remote sensing problems to conduct its classifications process. Likewise, the classifications of supervised images and classifications without supervision coupled with parametric and not parametric [18]. The classification of parametric and nonparametric images is based on pixels and fuzzy by using multiple applications in data analysis.

Two methods are used for unsupervised image classifications by using application [11], [21]. The application or software used can perform unknown pixel separation based on the bounce value in the classifications rather than by direction. The unattended method uses the techniques of K-Means and Iterative Self_organizing of Data Analysis or ISODATA.

Both methods are pixel-based and further process the statistical analysis based on spectral without considering the characteristics of the image [15], [31]. The Data used as an example can predict based on the variables measured. The depiction of the training area is represented by the most effective cover type when an image analyst has knowledge of geography for a region and experience in spectral class 
properties [18], [25]. Figure 4 shows the main steps in two common types of image classifications.

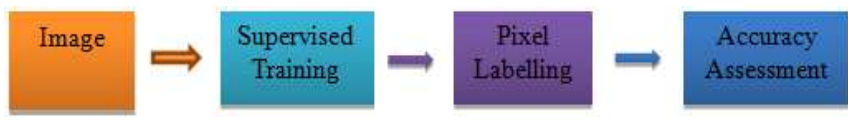

Fig.4 Supervised Image Classification Diagram

Figure 4 is the work of the supervised image classification method, which in the early stages of collecting the image dataset to do the training process with the supervised image classification method by marking the pixel label on the cloud layer Cumulonimbus to obtain accuracy value results. The research methodologies used in this study is illustrated in Figure 5

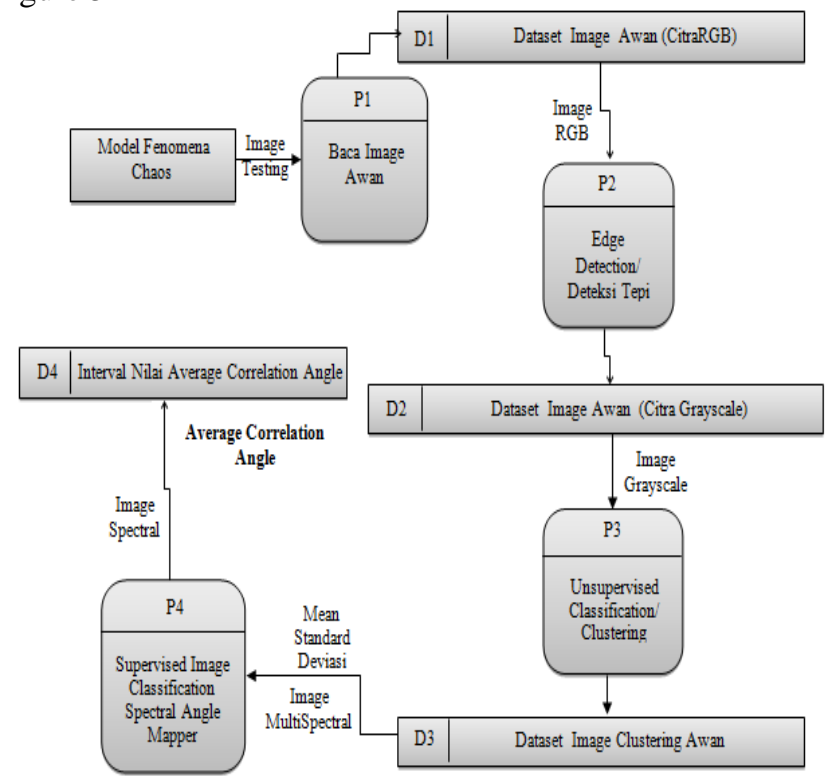

Fig.5 Research Methodology

Figure 5 shows four main processes. Process 1 (P 1) inputs cloud image, including the cumulus stage, clouds cirrus, clouds stratus, clouds cumulonimbus, and ordinary clouds. The cloud image is then saved to the cloud image dataset as an RGB image (D 1). After that, the process 2 (P2) uses an Edge detection algorithm to obtain a gray border of the image dataset testing which is the initials characteristic of the occurrence of the tornado. The image generated from the RGB cloud image conversion becomes the Grayscale cloud image. The result of a set of data testing is then saved as a Grayscale (D2) image. Process 1 (P1) and Process 2 (P 2) serve to extract an irregular pattern intensification on the cloud layer as well as to find out how fast the pixel intensity spreads to pixels (low to high frequencies). Once the image is extracted using the edge detection algorithm, next (P3) by performing Clustering or Unsupervised Classification process using the MultiSpecWin32 application. In this process, it serves to get the color intensity information of each pixel as well as to get the number of classes, the number of pixels, the standard deviation value, and the size of the classification contained in the cloud image dataset. One form of a cloud that is done by the process of the cluster is the image of cumulonimbus clouds. Then process 4 (P4) is using supervised image classification, the Spectral Angle Mapper algorithm, to get pixel sample count, percent, average correlation angle value, and the total amount of equal distance to class in pixels.

\section{RESULTS AND DISCUSSION}

The Model formed will be the testing process of the Cumulonimbus cloud dataset using the Edge Detection algorithm and Spectral Angle Mapper algorithm on Supervised Image Classification. It serves to test if the interval of value formed on the chaos phenomenon model Cumulonimbus Cloud $(\mathrm{Cb})$ to get the interval of the Average Correlation Angle value.

\section{A. Results Cumulonimbus Cloud Dataset Image using Edge Detection Algorithms}

In Figure 6 below as many as 24 cloud image data sets Cumulonimbus is a grayscale image. The image is the result of the extraction process using edge detection algorithms. This algorithm is used to obtain a gray edge border on a cumulonimbus cloud. The border is formed to facilitate the process of classifications..

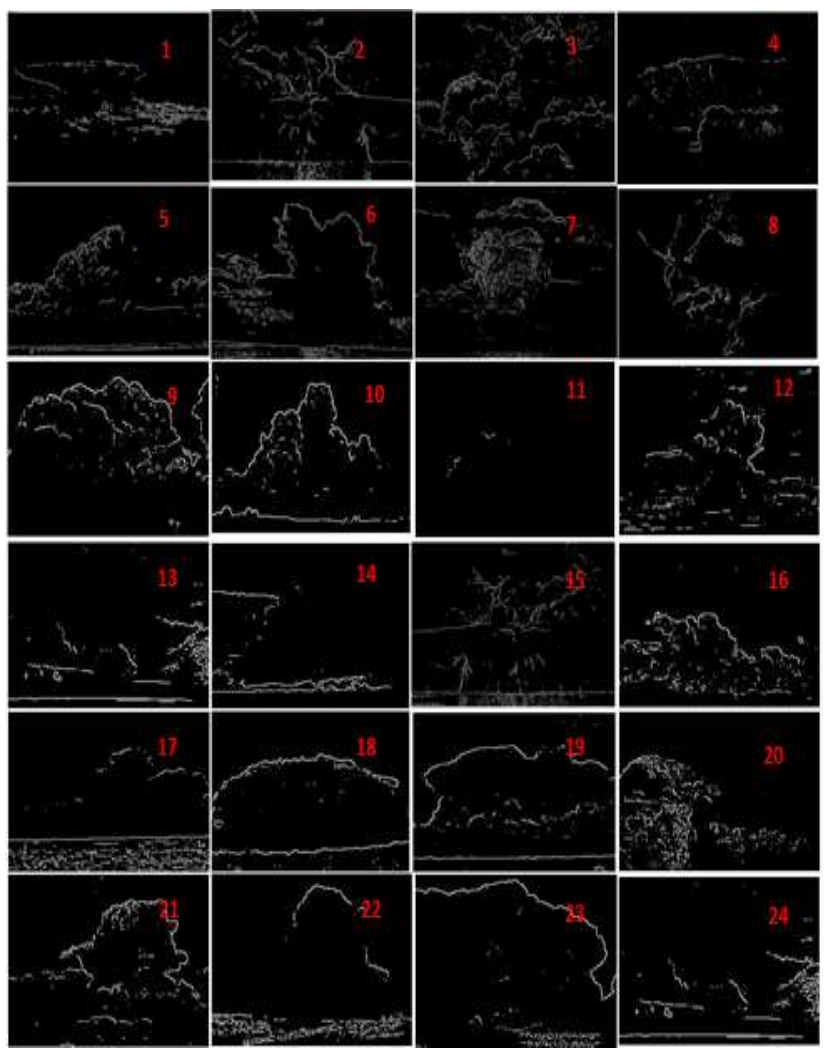

Fig.6 Grayscale Image Dataset

\section{B. Result of Cumulonimbus cloud datasets Images using Unsupervised Classification}

Once the extracted result is obtained, the clustering process is performed before using the supervised image classification method. This process aims to obtain a small number of classifications of pixel values and accelerate the classifications process time by using the supervised image classification method. The image dataset results are shown in figure 7. For statistical analysis result of mean and standard deviation values as found in Table I 


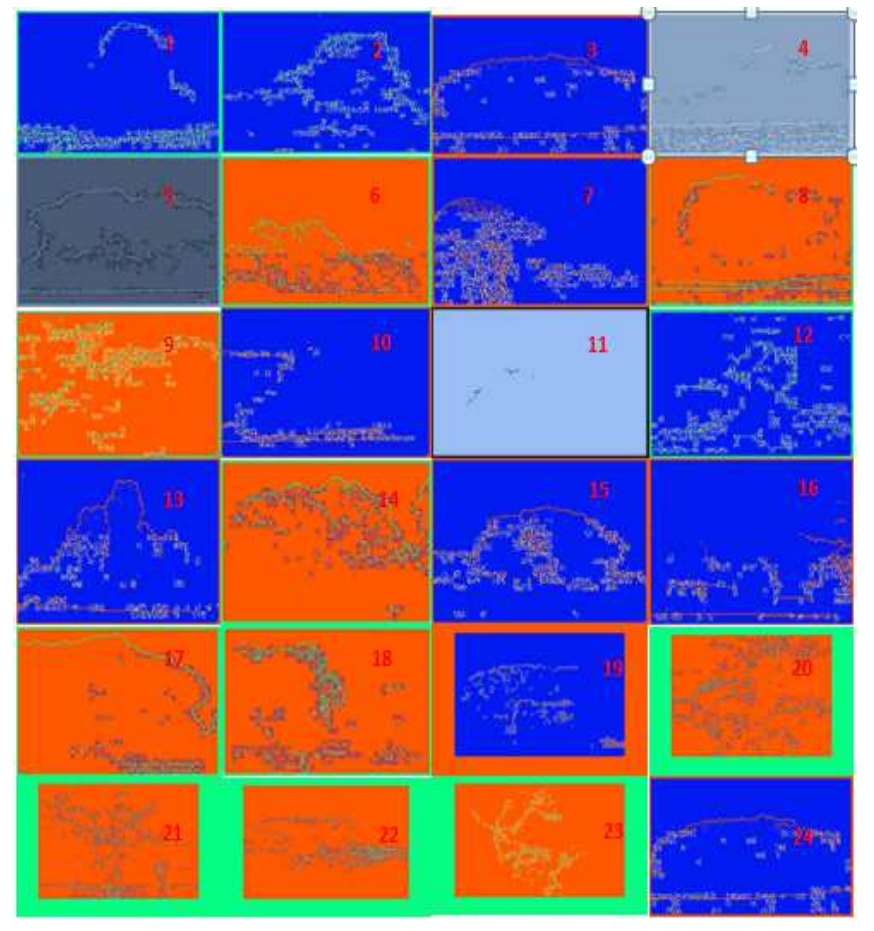

Fig.7 Dataset Image Clustering

In Figure 6 and Figure 7, the numbers 1 through 24, as many as 24 images produce grayscale, and the interval of Clustering values are at intervals $252.43-254.93$. In the results of clustering intervals of the pixel value 24 of the Cumulonimbus cloud image dataset, it has been initiated by signs of tornado events.

TABLE I

MEAN AND DEVIaTion STANDARd VAlues

\begin{tabular}{|c|c|c|c|c|c|c|}
\hline \multirow{2}{*}{ Image } & \multicolumn{2}{|c|}{ Maximum Mean Value } & \multicolumn{2}{c|}{$\begin{array}{c}\text { Maximum Deviation } \\
\text { Standard Value }\end{array}$} \\
\cline { 2 - 7 } & Red & Green & Blue & Red & Green & Blue \\
\hline Cb1 & 254.92 & 254.92 & 254.92 & 0.57 & 0.57 & 0.57 \\
\hline Cb2 & 254.91 & 254.91 & 254.91 & 0.62 & 0.62 & 0.62 \\
\hline Cb3 & 254.87 & 254.87 & 254.87 & 0.95 & 0.95 & 0.95 \\
\hline Cb4 & 254.88 & 254.88 & 254.88 & 0.94 & 0.94 & 0.94 \\
\hline Cb5 & 254.85 & 254.85 & 254.85 & 1.15 & 1.15 & 1.15 \\
\hline Cb6 & 254.81 & 254.81 & 254.81 & 1.32 & 1.32 & 1.32 \\
\hline Cb7 & 254.89 & 254.89 & 254.89 & 0.78 & 0.78 & 0.78 \\
\hline Cb8 & 254.93 & 254.93 & 254.93 & 0.51 & 0.51 & 0.51 \\
\hline Cb9 & 252.71 & 252.71 & 252.71 & 3.47 & 3.47 & 3.47 \\
\hline Cb10 & 252.68 & 252.68 & 252.68 & 4.02 & 4.02 & 4.02 \\
\hline $\mathrm{Cb} 11$ & 253.88 & 253.88 & 253.88 & 1.73 & 1.73 & 1.73 \\
\hline $\mathrm{Cb} 12$ & 253.88 & 253.88 & 253.88 & 1.62 & 1.62 & 1.62 \\
\hline $\mathrm{Cb} 13$ & 252.59 & 252.59 & 252.59 & 4.09 & 4.09 & 4.09 \\
\hline $\mathrm{Cb} 14$ & 252.98 & 252.98 & 252.98 & 3.57 & 3.57 & 3.57 \\
\hline $\mathrm{Cb} 15$ & 254.88 & 254.88 & 254.88 & 0.90 & 0.90 & 0.90 \\
\hline $\mathrm{Cb} 16$ & 252.95 & 252.95 & 252.95 & 3.52 & 3.52 & 3.52 \\
\hline $\mathrm{Cb} 17$ & 253.29 & 253.29 & 253.29 & 2.74 & 2.74 & 2.74 \\
\hline $\mathrm{Cb} 18$ & 252.78 & 252.78 & 252.78 & 3.78 & 3.78 & 3.78 \\
\hline $\mathrm{Cb} 19$ & 253.36 & 253.36 & 253.36 & 2.41 & 2.41 & 2.41 \\
\hline $\mathrm{Cb} 20$ & 252.70 & 252.70 & 252.70 & 3.82 & 3.82 & 3.82 \\
\hline $\mathrm{Cb} 21$ & 253.62 & 253.62 & 253.62 & 1.53 & 1.53 & 1.53 \\
\hline $\mathrm{Cb} 22$ & 253.67 & 253.67 & 253.67 & 1.64 & 1.64 & 1.64 \\
\hline $\mathrm{Cb} 23$ & 252.43 & 252.43 & 252.43 & 3.93 & 3.93 & 3.93 \\
\hline $\mathrm{Cb} 24$ & 252.80 & 252.80 & 252.80 & 3.65 & 3.65 & 3.65 \\
\hline
\end{tabular}

C. Result Dataset Image Cumulonimbus Clouds using the Spectral Angle Mapper Algorithm

The results obtained using the Spectral Angle Mapper algorithm are minimized and maximize intervals of the Average Correlation Angle pixel value with a value between $62.9^{\circ}-79.5^{\circ}$. The value can be seen in Table II.

TABLE II

AVERAge CoRrElation ANGLE VALUES

\begin{tabular}{|c|c|c|c|c|}
\hline Image & $\begin{array}{c}\text { Number } \\
\text { of } \\
\text { Clusters }\end{array}$ & Percent & $\begin{array}{c}\text { Average } \\
\text { Correlation } \\
\text { Angle }\end{array}$ & $\begin{array}{c}\text { Average } \\
\text { Correlation } \\
\text { Value }\end{array}$ \\
\hline $\mathrm{Cb} 1$ & 4 & 4.1 & 65.7 & 0.432 \\
\hline $\mathrm{Cb} 2$ & 4 & 36.8 & 64.0 & 0.438 \\
\hline $\mathrm{Cb} 3$ & 4 & 1.7 & 62.9 & 0.388 \\
\hline $\mathrm{Cb} 4$ & 4 & 1.59 & 66.5 & 0.425 \\
\hline $\mathrm{Cb} 5$ & 4 & 0.21 & 66.0 & 0.459 \\
\hline $\mathrm{Cb} 6$ & 3 & 0.035 & 75.9 & 0.407 \\
\hline $\mathrm{Cb} 7$ & 3 & 0.019 & 75.0 & 0.441 \\
\hline $\mathrm{Cb} 8$ & 4 & 0.012 & 72.8 & 0.41 \\
\hline $\mathrm{Cb} 9$ & 3 & 36.8 & 74.2 & 0.438 \\
\hline $\mathrm{Cb} 10$ & 4 & 1.7 & 49.1 & 0.388 \\
\hline $\mathrm{Cb} 11$ & 4 & 0.00095 & 73.2 & 0.438 \\
\hline $\mathrm{Cb} 12$ & 2 & 0.666 & 85.2 & 0.412 \\
\hline $\mathrm{Cb} 13$ & 3 & 2.86 & 79.5 & 0.415 \\
\hline $\mathrm{Cb} 14$ & 4 & 2.999 & 72.0 & 0.423 \\
\hline $\mathrm{Cb} 15$ & 4 & 0.179 & 73.8 & 0.395 \\
\hline $\mathrm{Cb} 16$ & 3 & 1.593 & 71.7 & 0.425 \\
\hline $\mathrm{Cb} 17$ & 4 & 0.035 & 72.6 & 0.407 \\
\hline $\mathrm{Cb} 18$ & 4 & 0.019 & 73.7 & 0.441 \\
\hline $\mathrm{Cb} 19$ & 4 & 0.21 & 74.6 & 0.459 \\
\hline $\mathrm{Cb} 20$ & 3 & 1.7 & 76.2 & 0.388 \\
\hline $\mathrm{Cb} 21$ & 4 & 0.012 & 74.0 & 0.41 \\
\hline $\mathrm{Cb} 22$ & 4 & 0.179 & 76.5 & 0.395 \\
\hline $\mathrm{Cb} 23$ & 4 & 2.86 & 77.4 & 0.415 \\
\hline $\mathrm{Cb} 24$ & 4 & 4.1 & 62.9 & 0.432 \\
\hline
\end{tabular}

Table I and Table II are the results of the Supervised Image Classification analysis with the Spectral Angle Mapper algorithm. The resulting analysis is to get the maximum average value and standard deviation for the RGB color spectrum.

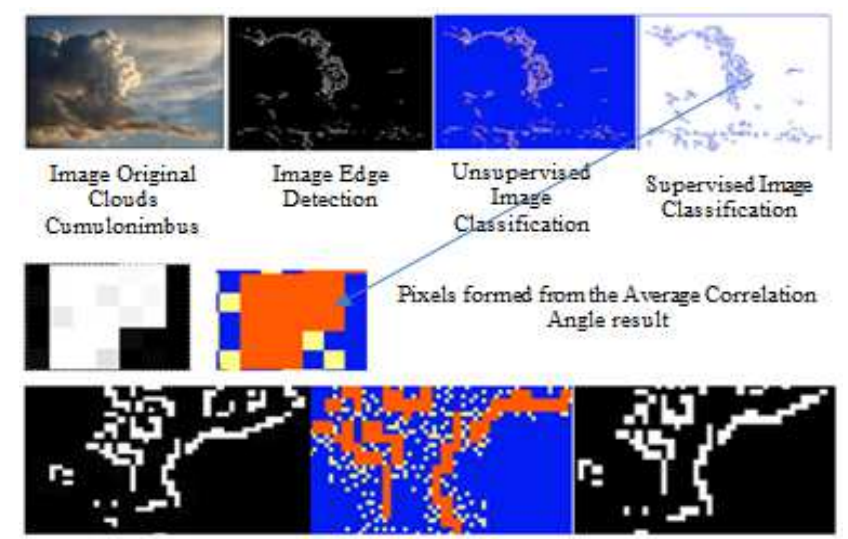

Fig.8 Illustration Spectral Angle Mapper

An illustration or overview of the algorithm determination obtained until the result of a spectral angle is shown in Figure 8 . The spectral angle aims to get the intensity interval of the Average Correlation Angle pixel value between the layers contained in the cumulonimbus cloud image. In the Spectral Angle Mapper method, the darker pixels of the 
output show a smaller spectral angle. Smaller angles show a resemblance between a spectral reference and a spectral test. The inputs used in the SAM algorithm implemented in the software are the Cumulonimbus cloud dataset.

\section{IV.CONCLUSION}

The final result of the classification supervised image process is at the intensities of the minimized and maximum pixel values for the average correlation angle. Findings from 24 dataset of Cumulonimbus Cloud are as follows:

Table I. is a result of processing clustering (unsupervised classification) before doing the classifications process (Supervised Classification) using a Single Pass algorithm. These are the result of the cloud Cumulonimbus dataset used to obtain the minimized and maximum pixel value intervals. Pictures Number 1 to 24 show the result of mean value, standard deviation, and maximum classification size. Table II shows the minimized and maximized values of the average correlation angle which are the final results using the supervised image classification method.

Reviewed from the number of clusters that formed an average of 4 clusters are as many as 17 datasets, 3 clusters of 6 datasets and 2 clusters of only 1 dataset. The number of pixels, the mean value, the standard deviation value, and the classification size, results in the intensity interval of the maximum pixel value are located at the number of sample cluster 4 in the dataset at the interval of pixel value of $252.43-254.93$ and the standard deviation value of $0.51-$ 4.09 , for the maximize classification of the average of 155.766 and the interval of Average Correlation Angle value of between $62.9^{\circ}-79.5^{\circ}$. The value of accuracy obtained from 24 data cloud cumulonimbus using the supervised image classification algorithm based on the average angle of correlation is $95.83 \%$.

\section{ACKNOWLEDGMENT}

This research is part of dissertation research. Hence, this paper is under supervision of the dissertation supervisors of computer Science study Program, University of North Sumatra. This research is also supported by Universitas Asahan. This paper is subject to open hearing as a requirement for doctoral program accomplishment.

\section{REFERENCES}

[1] J. Alvarez-Ramirez, C. Ibarra-Valdez, and E. Rodriguez, "Fractal analysis of Jackson Pollock's painting evolution," Chaos, Solitons and Fractals, vol. 83, pp. 97-104, 2016.

[2] J. P. Cangialosi, A. S. Latto, and R. Berg, "National Hurricane Center Tropical Cyclone Report - Hurricane Irma (AL112017) 30 August -12 September 2017," no. September 2017, p. 111, 2018.

[3] J. P. Cangialosi and J. L. Franklin, "2014 National Hurricane Center Forecast Verification Report John P. Cangialosi and James L. Franklin NOAA/NWS/NCEP/National Hurricane Center 25 March 2015," no. March, pp. 1-82, 2015.

[4] S. Banerjee, "An Algorithm for Pre-Processing of Satellite Images of Cyclone Clouds An Algorithm for Pre-Processing of Satellite Images of Cyclone Clouds," no. September 2013, 2016.

[5] M. Infromasi et al., "Jurnal Geografi," vol. 12, no. 2, pp. 143-154, 2015.

[6] V. Wiley and T. Lucas, "Computer Vision and Image Processing: A Paper Review," vol. 2, no. 1, pp. 28-36, 2018.

[7] R. DeMaria, "Automated Tropical Cyclone Eye Detection Using Submitted by," 2015
[8] F. Liantoni, N. Suciati, and C. Fatichah, "Modifikasi Ant Colony Optimization Berdasarkan Gradient Untuk Deteksi Tepi Citra," pp. 225-234, 2015.

[9] C. Wang, A. Xu, and X. Li, "Supervised classification highresolution remote-sensing image based on interval type-2 fuzzy membership function," Remote Sens., vol. 10, no. 5, 2018.

[10] L. Ma, M. Li, X. Ma, L. Cheng, P. Du, and Y. Liu, "A review of supervised object-based land-cover image classification," ISPRS J. Photogramm. Remote Sens., vol. 130, pp. 277-293, 2017.

[11] I. Kahraman, M. K. Turan, and I. R. Karas, "Road Detection from High Satellite Images Using Neural Networks," vol. 5, no. 4, pp. 304-307, 2015.

[12] Jingying Tang and Corene Matyas, "A Nowcasting Model for Tropical Cyclone Precipitation Regions Based on the TREC Motion Vector Retrieval with a Semi-Lagrangian Scheme for Doppler Weather Radar," 2018.

[13] A. Setiyoko et al., "Suitability Analysis of Remote Sensing Data Services Against User Needs," Semin. Nas. Penginderaan Jauh 2016, pp. 424-527, 2016.

[14] F. Collins, M. M. Branch, F. Collins, and F. Collins, "A Climatology of Hurricane Eye Formation *," no. Dvorak 1984, pp. 1405-1426, 2012.

[15] E. Dong, G. Zhu, C. Chen, J. Tong, Y. Jiao, and S. Du, "Introducing chaos behavior to kernel relevance vector machine (RVM) for fourclass EEG classification," PLoS One, vol. 13, no. 6, pp. 1-19, 2018.

[16] D. Camilo, D. O. Duarte, J. Zanetti, and J. G. Junior, "Comparison of supervised classification methods of Maximum Likelihood image , Minimum Distance, Parallelepiped and Neural network in images of Unmanned Air Vehicle ( UAV ) in," Proc. XVII GEOINFO, no. 2004, pp. 12-21, 2016.

[17] D. J. Wang, X. Shi, D. A. McFarland, and J. Leskovec, "Measurement error in network data: A re-classification," Soc. Networks, vol. 34, no. 4, pp. 396-409, 2012.

[18] R. Sirefelt, "Road Extraction from Aerial Images," 2015

[19] J. St. Peter, J. Hogland, N. Anderson, J. Drake, and P. Medley, "Fine Resolution Probabilistic Land Cover Classification of Landscapes in the Southeastern United States," ISPRS Int. J. Geo-Information, vol. 7, no. 3, p. 107,2018

[20] A. Environmentally, F. Tropical, and C. Hazard, "Journal of Advances in Modeling Earth Systems," pp. 223-241, 2018.

[21] L. Metz, N. Maheswaranathan, B. Cheung, and J. Sohl-Dickstein, "Learning Unsupervised Learning Rules," 2018.

[22] C. Peng, D. Yue, and M. R. Fei, "Relaxed stability and stabilization conditions of networked fuzzy control systems subject to asynchronous grades of membership," IEEE Trans. Fuzzy Syst., vol. 22, no. 5, pp. 1101-1112, 2014.

[23] Indarto, "Penginderaan Jauh: Metode Analisis \& Interpretasi Citra Satelit," no. June, 2017.

[24] A. Peytchev, A. Peytchev, and E. Peytcheva, "Reduction of Measurement Error due to Survey Length: Evaluation of the Split Questionnaire Design Approach," Surv. Res. Methods, vol. 11, no. 4, pp. 361-368, 2017.

[25] A. Zhang and Y. Xie, "Chaos theory-based data-mining technique for image endmember extraction: Laypunov index and correlation dimension (L and D)," IEEE Trans. Geosci. Remote Sens., vol. 52, no. 4, pp. 1935-1947, 2014.

[26] C. Eyupoglu, M. A. Aydin, A. H. Zaim, and A. Sertbas, "An Efficient Privacy Preserving Algorithm Based on Chaos and Perturbation in Big Data," pp. 1-19, 2018.

[27] A. D. Hidayat and I. Afrianto, "Sistem Kriptografi Citra Digital pada Jaringan Intranet Menggunakan Metode Kombinasi Chaos Map dan Teknik Selektif," Ultimatics, vol. 9, no. 1, pp. 59-66, 2017.

[28] C. Chen, J. Liu, and J. Syu, "Application of Chaos Theory and Data Mining to Seizure Detection of Epilepsy," Dimens. Contemp. Ger. Arts Lett., vol. 25, pp. 23-28, 2012.

[29] S. Mangiarotti, F. Le Jean, M. Huc, and C. Letellier, "Global modeling of aggregated and associated chaotic dynamics," Chaos, Solitons and Fractals, vol. 83, pp. 82-96, 2016.

[30] Y. Shen, "Improved chaos genetic algorithm based state of charge determination for lithium batteries in electric vehicles," Energy, vol. 152, pp. 576-585, 2018.

[31] S. Mishra, "Supervised classification of dermatological diseases via Deep learning," 2018 